\title{
SCIENTIFIC REPORTS

\section{Association between Fatty Liver Index and Periodontitis: the Korea National Health and Nutrition Examination Survey}

\author{
Ji-Youn $\mathrm{Kim}^{1}$, Gyu-Na Lee ${ }^{2}$, Hyun Chul Song ${ }^{1}$, Yong-Moon Park $\mathbb{D}^{3}$, Yu-Bae Ahn ${ }^{4}$, \\ Kyungdo Han ${ }^{2 *}$ \& Seung-Hyun $\mathrm{Ko}^{4 *}$
}

It has been suggested that periodontitis is associated with metabolic abnormalities including nonalcoholic fatty liver disease (NAFLD). The fatty liver index (FLI) is a non-invasive surrogate marker and predictor of NAFLD. We aimed to determine whether FLI itself would be associated with periodontitis through a secondary analysis of previously reported nationally representative probability sample data of the Korean population. FLI was calculated from a previously developed algorithm which combines measures of body mass index (BMI), waist circumference, triglyceride, and gamma-glutamyl transferase (GGT). Periodontitis was diagnosed based on the Community Periodontal Index (CPI) developed by the World Health Organization. Of 4,272 participants, $26.1 \%$ were diagnosed with periodontitis. Higher FLI was associated with a higher prevalence of periodontitis (Odds ratio (OR) highest vs. Iowest quartile of $\mathrm{FL}, 1.63 ; 95 \%$ confidence interval ( $\mathrm{Cl}), 1.23-2.16 ; \mathrm{P}=0.001$ for trend) adjusting for confounding factors. In the highest FLI quartile, prevalence of periodontitis was higher in individuals

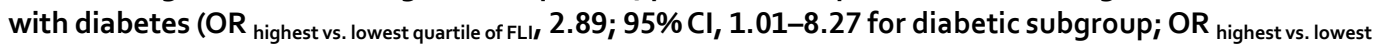
quartile of $\mathrm{FLI}, 1.45 ; 95 \% \mathrm{Cl}, 1.07-1.96$ for non-diabetic subgroup). In summary, FLI was associated with prevalent periodontitis.

Periodontitis is a dental bacteria-induced chronic inflammatory condition characterized by immunologic destruction of supporting structures of teeth ${ }^{1}$. Approximately half of the adult U.S. population suffers from periodontitis with data from the 2009 and 2010 National Health and Nutrition Examination Survey ${ }^{2}$. Associations between periodontitis and other health problems including obesity, coronary heart disease, stroke, metabolic syndrome, diabetes mellitus, and non-alcoholic fatty liver disease (NAFLD) have been already discovered ${ }^{3-8}$.

Fatty liver index (FLI) is an algorithm that combines measures of body mass index (BMI), waist circumference, triglyceride, and gamma-glutamyl transferase $(\mathrm{GGT})^{9}$. FLI is a non-invasive surrogate marker and predictor of NAFLD ${ }^{8,9}$. For the definitive diagnosis of NAFLD, the ultrasonic evaluation or liver biopsy is required, while FLI may allow us to identify NAFLD in large-scale epidemiological research.

We hypothesize that FLI may be associated with periodontitis prevalence. Therefore, the objective of this study was to assess the relationship between FLI and periodontitis in a large probability sample of the Korean population using data from the 2010 Korea National Health and Nutrition Examination Survey (KNHANES). Since NAFLD accounts for $20-30 \%$ and $5-18 \%$ of the general population in Western and Asian countries ${ }^{10}$, respectively, it is assumable that a population group with a high prevalence of periodontitis may exist among those not diagnosed with NAFLD but possess high FLI. This hypothesis may be observable in a large population dataset such as KNHANES. Benefitting from the size of the dataset, we stratified the Korean general population according to FLI quartiles to see if the association with periodontitis was stronger in subjects with higher FLI.

\footnotetext{
${ }^{1}$ Division of Oral \& Maxillofacial Surgery, Department of Dentistry, St. Vincent's Hospital, College of Medicine, The Catholic University of Korea, 222 Banpo-daero, Seocho-gu, Seoul, 06591, Republic of Korea. ${ }^{2}$ Statistics and Actuarial Science, Soongsil University, 369 Sangdo-ro, Dongjak-gu, Seoul, 06978, Republic of Korea. ${ }^{3}$ Epidemiology Branch, National Institute of Environmental Health Sciences, National Institutes of Health, Research Triangle Park, 111T. W. Alexander Dr., Research Triangle Park, Durham, NC, 27709, USA. ${ }^{2}$ Division of Endocrinology and Metabolism, Department of Internal Medicine, St. Vincent's Hospital, College of Medicine, The Catholic University of Korea, 222 Banpo-daero, Seocho-gu, Seoul, 06591, Republic of Korea. *email: hkd917@naver.com; kosh@catholic.ac.kr
} 


\begin{abstract}
Methods
Survey and subjects. Data of this study were obtained by secondary analysis of a previously reported nation-wide probability sample data, which was previously reported as the 2010 Korea National Health and Nutrition Examination Survey (KNHANES) study ${ }^{11}$. This survey was conducted by the Division of Chronic Disease Surveillance, Korea Centers for Disease Control and Prevention under Korean Ministry of Health and Welfare, Sejong, Korea ${ }^{12}$. Trained interviewers collected nationally representative samples based on standard household surveys ${ }^{12}$. From sampled household units based on population and housing census from the 2005 National Census Registry in Korea, participants were selected by using a stratified, multistage, and probability-based sampling design with proportional allocation ${ }^{13}$. A total of 8,473 participants were candidates in the 2010 KNHANES survey. For this study, 6352 participants aged over 19 years were included. After excluding those with cancer, liver diseases, low kidney function, pregnancy, heavy drinking, or missing data, 4,272 participants were analyzed. Informed consent was obtained from all interviewees in this survey. This study was approved by the Institutional Review Board (IRB) of the Korean Center for Disease Control and Prevention (IRB number: 2010-02CON-21-C) and was conducted according to Declaration of Helsinki guidelines. Informed consent was obtained from all participants at the time of survey collection.
\end{abstract}

Sociodemographic and lifestyle variables. A self-administered questionnaire was used to assess sociodemographic and lifestyle variables (including smoking, alcohol consumption, physical exercise, household income, and education level) of participants. Regarding smoking status, participants were classified as current smokers or nonsmokers. Those who smoked currently and had smoked more than 100 cigarettes in their lifetime were defined as current smokers ${ }^{14}$. International physical activity questionnaire was used to measure the degree of physical exercise. Regular exercisers were defined as those who exercised for $\geq$ five occasions per week for 30 minutes per session or those who participated in strenuous physical activity for $\geq$ three occasions per week for 20 minutes per session ${ }^{14,15}$. Education level was categorized as above (high school education or above) or less (middle school education or less) than ten years of education. Household income was divided by the number of included family members and categorized into quartiles. The lowest quartile of household income was $<$ USD $1,092.40$ per month.

Anthropometric and biochemical measurements. Anthropometric measurements of study participants were taken by trained staff members. Bodyweight and height were measured for subjects with indoor clothing without shoes. For waist circumference, the narrowest point between the iliac crest and the lower border of the rib cage was used. Body mass index (BMI) was calculated using the formula of weight $/$ height ${ }^{2}\left(\mathrm{~kg} / \mathrm{m}^{2}\right)$. For systolic and diastolic blood pressure measurements, a standard mercury sphygmomanometer (Braumanometer; W.A. Baum Co., Inc., Copiague, NY, USA) was used. The measurement was done twice at a 5-minute interval. Venous blood was collected from the antecubital vein of each participant after a fasting period of 8 hours. Concentrations of fasting plasma glucose, total cholesterol, triglycerides, high-density lipoprotein (HDL) cholesterol, low-density lipoprotein (LDL) cholesterol, and GGT were measured from blood samples using an automated chemistry analyzer (Hitachi Automatic Analyzer 7600, Hitachi, Tokyo, Japan) and commercially available kits (Daiichi, Tokyo, Japan).

Diabetes was defined as fasting glucose higher than $126 \mathrm{mg} / \mathrm{dL}$, current use of anti-diabetic medication, or previous physician diagnosis of diabetes. Metabolic syndrome was defined if three or more of the following criteria were fulfilled ${ }^{16}$ : waist circumference of $\geq 90 \mathrm{~cm}$ in men and $\geq 85 \mathrm{~cm}$ in women; fasting triglyceride $\geq 150 \mathrm{mg} /$ $\mathrm{dL}$ or the use of lipid-lowering medication; HDL cholesterol $<40 \mathrm{mg} / \mathrm{dL}$ in men and $<50 \mathrm{mg} / \mathrm{dL}$ in women or the use of associated medication; blood pressure of $130 / 85 \mathrm{mmHg}$ or higher or current use of associated medication. Diabetes mellitus, one of obesity-related metabolic disorders that affects nearly $10 \%$ of world population ${ }^{17}$, is characterized by hyperglycemia caused by defects in insulin secretion, insulin action, or both ${ }^{18}$.

FLI is a noninvasive method of assessing hepatic steatosis predicted with body mass index (BMI), waist circumference, triglyceride, and gamma-glutamyl transferase (GGT), calculated with the following formula9:

$$
\begin{aligned}
& \text { FLI }=\left(\mathrm{e}^{0.953 \times \log (\text { triglycerides })+0.139 \times \mathrm{BMI}+0.718 \times \operatorname{loge}(\mathrm{GGT})+0.053 \times \text { waistcircumference }-15.745}\right) / \\
& \left(1+\mathrm{e}^{0.953 \times \log e(\text { triglycerides })+0.139 \times \mathrm{BMI}+0.718 \times \operatorname{loge}(\mathrm{GGT})+0.053 \times \text { waistcircumference }-15.745}\right) \times 100
\end{aligned}
$$

$\mathrm{BMI}$ is a determinant of obesity, with BMI $>30 \mathrm{~kg} / \mathrm{m}^{2}$ being considered to be obese ${ }^{19}$. BMI is an indicator of body fat accumulation. However, it is not indicative of fat distribution. Therefore, sometimes waist circumference is used in combination with BMI when defining obesity ${ }^{3}$. Triglycerides are main constituents of body fat. They are proportionally related to obesity ${ }^{20}$. GGT is an enzyme found in many tissues, especially abundant in the liver ${ }^{21}$. Blood GGT concentration is a sensitive biomarker of obesity. It is known to be increased in obesity-related health problems such as metabolic syndrome, diabetes, hypertension, dyslipidemia, and cardiovascular diseases ${ }^{22}$.

Subjects were divided into quartiles according to the value of FLI, each of male and female. The cut-off values in 1st quartile are $12.4 \mathrm{in}$ male $(\mathrm{n}=376)$ and $4.6 \mathrm{in}$ female $(\mathrm{n}=691)$. The cut-off values in 2 nd quartile are $26.6 \mathrm{in}$ male $(n=377)$ and 10.7 in female $(n=692)$. The cut-off values in 3rd quartile are $49.6 \mathrm{in}$ male $(\mathrm{n}=377)$ and 26.7 in female $(n=692)$. And the range of values in 4 th quartile are above 49.6 to 99.5 in male $(n=376)$ and above 26.7 to 95.6 in female $(n=691)$.

Oral health behaviors and periodontal status. Daily toothbrushing frequency was included in the questionnaire. The survey also included history of a periodic dental checkup categorized into yes (at least once during the past year) or no. Community periodontal index (CPI) developed by the World Health Organization was utilized to evaluate the presence of periodontal disease ${ }^{23}$. Index teeth were $11,16,17,26,27,31,36,37,46$, 47 according to the Federation Dentaire Internationale system. Periodontitis was defined as CPIs of 3 and 4 . CPI 
3 was given to cases with a shallow pocket (depth of 3.5-5.5 mm) from at least one site in one of the index teeth while CPI 4 was considered for cases with a deep pocket (depth $\geq 5.5 \mathrm{~mm}$ ).

Statistical analysis. Results are presented as means \pm standard error or percentages (standard error). For the variables which did not follow the normal distribution, logarithmic transformation was performed, and the results are presented as geometric mean $\pm 95 \%$ confidence intervals $(\mathrm{CI})$. The relationship between values was obtained by independent t-test for continuous variables or chi-square test for categorical variables using SURVEYMEANS or SURVEYREQ procedures. Multiple logistic regression analysis was used to evaluate the association of periodontitis with FLI using SURVEYLOGISTIC procedure. In the sub-analysis on diabetes mellitus, the quartile of FLI was same as the full model. Odds ratios (OR) and 95\% CI were calculated after adjusting for potential confounders. Cofounders included in Model 1 were age and sex. Model 2 was adjusted for variables in Model 1 plus smoking, exercise, drinking, income, and education. Model 3 was adjusted for variables in Model 2 plus frequency of tooth brushing and history of a periodic dental checkup. Log transformation was applied when appropriate. Statistical analysis was performed using SAS version 9.4 (SAS Institute, Inc., Cary, NC) to account for the complex sampling design ${ }^{24}$. Two-sided $\mathrm{P}$ values of $<0.05$ was considered a statistically significant difference.

\section{Results}

Baseline characteristics of subjects with periodontitis. Of 4,272 participants, 1,113 were diagnosed with periodontitis. Table 1 details baseline characteristics of participants included in the study population by the presence of periodontitis. Periodontitis subjects were older than control subjects ( 53.1 vs. 41.2 years, $P<0.0001$ ). They also had higher percentage of males $(51.7 \%$ vs. $38.9 \%, P<0.0001)$. Regarding anthropometric factors associated with obesity, the periodontitis group showed higher BMI (24.2 vs. 23.3, $P<0.0001)$, larger waist circumference $(83.4 \mathrm{~cm}$ vs. $78.9 \mathrm{~cm}, P<0.0001)$, higher systolic blood pressure $(124.9 \mathrm{mmHg}$ vs. $115.9 \mathrm{mmHg}, P<0.0001)$, and higher diastolic blood pressure $(79 \mathrm{mmHg}$ vs. $75.7 \mathrm{mmHg}, P<0.0001)$. When obesity-related laboratory indices were considered, the periodontitis group had higher glucose level $(102.6 \mathrm{mg} / \mathrm{dL}$ vs. $93.2 \mathrm{mg} / \mathrm{dL}, P<0.0001)$, higher triglyceride level $(120.5 \mathrm{mg} / \mathrm{dL}$ vs. $95.7 \mathrm{mg} / \mathrm{dL}, P<0.0001)$, higher total cholesterol level $(192.6 \mathrm{mg} / \mathrm{dL}$ vs. $183.5 \mathrm{mg} / \mathrm{dL}, P<0.0001)$, higher LDL level $(118.3 \mathrm{mg} / \mathrm{dL}$ vs. $111.6 \mathrm{mg} / \mathrm{dL}, P<0.0001)$, but lower HDL level $(45.8 \mathrm{mg} / \mathrm{dL}$ vs. $48.9 \mathrm{mg} / \mathrm{dL}, P<0.0001)$. Serum glutamic oxaloacetic transaminase level $(22.3 \mathrm{U} / \mathrm{L}$ vs. $20.7 \mathrm{U} / \mathrm{L}$, $P<0.0001)$ and glutamic pyruvic transaminase level $(22.4 \mathrm{U} / \mathrm{L}$ vs. $20.4 \mathrm{U} / \mathrm{L}, P=0.0007)$ were higher in the periodontitis group than those in the control group. Percentage of subjects having metabolic syndrome $(42.1 \%$ vs. $21.6 \%, P<0.0001)$ or diabetes $(15.6 \%$ vs. $5.5 \%, P<0.0001)$ was also higher in the periodontitis group than that in the control group. Additionally, individuals with periodontitis were more likely to be a current smoker $(25.6 \%$ vs. $19.2 \%, P=0.002)$, of the lowest quartile income $(22.7 \%$ vs. $14.2 \%, P<0.0001)$, and less educated $(52.8 \%$ vs. $78.2 \%, P<0.0001)$. However, alcohol consumption $(P=0.2539)$ and regular physical exercise $(P=0.9319)$ were similar between the periodontitis group and the control group. The periodontitis group had less frequent toothbrushing $(P=0.001)$ and less percentage of periodontal checkup during the past year $(18.2 \%$ vs. $23.7 \%$, $P=0.001)$. FLI was significantly higher in individuals with periodontitis $(21.6$ vs. $12.2, P<0.0001)$.

FLI-related characteristics of subjects with periodontitis. Table 2 compares prevalence and control of periodontitis according to variables used to calculate FLI. The periodontitis group had more BMI-high population $\left(\geq 30 \mathrm{~kg} / \mathrm{m}^{2}\right)$ than the control group $(5.2 \%$ vs. $3.5 \%, P<0.0001)$ but less BMI-low $\left(<18.5 \mathrm{~kg} / \mathrm{m}^{2}\right)$ population ( $2.8 \%$ vs. $5.7 \%, P<0.0001)$. Percentage of population with waist circumstances above the cut-off for metabolic syndrome was higher in subjects with periodontitis than that in control subjects $(40.4 \%$ vs. $27.6 \%, P<0.0001)$. Similarly, percentage of those with triglyceride levels above cut-off for metabolic syndrome was higher in the periodontitis group than that in the control group $(37.2 \%$ vs. $23.8 \%, P<0.0001)$. The periodontitis group had higher percentage of those with GGT-high (highest quartile) (35\% vs. $23.3 \%, P<0.0001$ ) than the control group, although it had lower percentage of those with GGT-low (lowest quartile) $(12.6 \%$ vs. $25.2 \%, P<0.0001)$ than the control group. Baseline characteristics of the study population according to FLI quartiles are additionally described in detail in Supplementary Table 1.

Adjusted ORs of periodontitis according to FLI quartiles. Table 3 shows adjusted OR and their 95\% CI from multiple logistic regression analyses for subjects with periodontitis according to FLI quartiles. After adjusting for sex and age (Model 1), adjusted OR (95\% CI) was 1.731 (1.317-2.292) in the highest quartile of FLI compared to the lowest quartile. When current smoking status, regular exercise, alcohol consumption, income, and education level were further controlled (Model 2), the adjusted OR (95\% CI) was $1.664(1.267-2.185)$ in the highest quartile of FLI compared to the lowest quartile. With additional adjustment for toothbrushing frequency and periodontal checkup (Model 3), the adjusted OR (95\% CI) was 1.632 (1.235-2.185) in the highest quartile of FLI compared to the lowest quartile.

Adjusted ORs of periodontitis according to FLI quartiles in the diabetes subgroup. The association of diabetes with periodontitis has been extensively studied ${ }^{25}$. Since higher prevalence of diabetes mellitus in the periodontitis group was demonstrated in our data (Table 1), we performed additional multiple logistic regression analyses on subgroups with or without diabetes mellitus. In the non-diabetes subgroup, adjusted ORs (95\% CIs) in the highest quartile of FLI compared to the lowest quartile by Model 1, Model 2, and Model 3 were 1.545 (1.142-2.090), 1.470 (1.090-1.981), and 1.448 (1.068-1.963), respectively. In the diabetes subgroup, adjusted ORs ( $95 \%$ CIs) in the highest quartile of FLI compared to the lowest quartile by Model 1, Model 2, and Model 3 were $2.724(0.954-7.774), 2.936(1.069-8.068)$, and 2.891 (1.018-8.269), respectively.

Severity of periodontitis according to FLI quartiles in the diabetes subgroup. Table 4 shows percentages of CPI 3 and CPI 4 periodontitis categorized by FLI quartiles. In the total study population, percentages 


\begin{tabular}{|c|c|c|c|}
\hline & \multicolumn{2}{|l|}{ Periodontitis } & \multirow[b]{3}{*}{$\mathbf{P}$} \\
\hline & \multirow{2}{*}{$\begin{array}{l}\text { No } \\
\mathrm{n}=3159\end{array}$} & \multirow{2}{*}{\begin{tabular}{|l|} 
Yes \\
$n=1113$ \\
\end{tabular}} & \\
\hline & & & \\
\hline Age (years) & $41.2 \pm 0.5$ & $53.1 \pm 0.6$ & $<0.0001$ \\
\hline Sex (male) & $38.9(1.1)$ & $51.7(1.6)$ & $<0.0001$ \\
\hline Body mass index $\left(\mathrm{kg} / \mathrm{m}^{2}\right)$ & $23.3 \pm 0.1$ & $24.2 \pm 0.1$ & $<0.0001$ \\
\hline Waist circumference $(\mathrm{cm})$ & $78.9 \pm 0.3$ & $83.4 \pm 0.4$ & $<0.0001$ \\
\hline Systolic blood pressure (mmHg) & $115.9 \pm 0.4$ & $124.9 \pm 0.9$ & $<0.0001$ \\
\hline Diastolic blood pressure (mmHg) & $75.5 \pm 0.3$ & $79 \pm 0.5$ & $<0.0001$ \\
\hline Metabolic syndrome (\%) & $21.6(0.9)$ & $42.1(2)$ & $<0.0001$ \\
\hline Diabetes mellitus (\%) & $5.5(0.4)$ & $15.6(1.6)$ & $<0.0001$ \\
\hline Hypertension (\%) & $21.1(0.9)$ & $41.9(2.3)$ & $<0.0001$ \\
\hline Current smoking (\%) & $19.2(0.9)$ & $25.6(1.5)$ & 0.0002 \\
\hline Alcohol consumption (\%) ${ }^{\mathrm{a}}$ & $53(1.3)$ & $50.6(1.8)$ & 0.2539 \\
\hline Regular exercise $(\%)^{\mathrm{b}}$ & $22.7(1.2)$ & $22.8(1.8)$ & 0.9319 \\
\hline Low income (lowest quartile \%) & $14.2(1)$ & $22.7(2)$ & $<0.0001$ \\
\hline Low education (below high school \%) & $21.8(1.2)$ & $47.2(2.7)$ & $<0.0001$ \\
\hline Tooth brushing frequency (\%) & & & $<0.0001$ \\
\hline Once a day & $8.2(0.7)$ & $16.9(1.9)$ & \\
\hline Twice a day & $46.8(1.2)$ & $51.4(2.1)$ & \\
\hline Thrice a day & $45(1.3)$ & $31.6(2.1)$ & \\
\hline Periodic dental checkup $(\%)^{c}$ & $23.7(1.2)$ & $18.2(1.6)$ & 0.0014 \\
\hline \multicolumn{4}{|l|}{ Biochemical measurements } \\
\hline Glucose (mg/dL) & $93.2 \pm 0.4$ & $102.6 \pm 1.5$ & $<0.0001$ \\
\hline Triglycerides (mg/dL) & $95.7(93.3-98.1)$ & $120.5(115.9-125.3)$ & $<0.0001$ \\
\hline Total cholesterol (mg/dL) & $183.5 \pm 0.9$ & $192.6 \pm 1.2$ & $<0.0001$ \\
\hline LDL cholesterol (mg/dL) & $111.6 \pm 0.8$ & $118.3 \pm 1.2$ & $<0.0001$ \\
\hline HDL cholesterol (mg/dL) & $48.9 \pm 0.2$ & $45.8 \pm 0.4$ & $<0.0001$ \\
\hline Gamma-glutamyl transferase $(\mathrm{mg} / \mathrm{dL})$ & $21.5(20.9-22.1)$ & $26.7(25.6-27.9)$ & $<0.0001$ \\
\hline Glutamic oxaloacetic transaminase (U/L) & $20.7 \pm 0.3$ & $22.3 \pm 0.3$ & $<0.0001$ \\
\hline Glutamic pyruvic transaminase (U/L) & $20.4 \pm 0.4$ & $22.4 \pm 0.5$ & 0.0007 \\
\hline Fatty Liver Index & $12.2(11.5-12.9)$ & $21.6(20.1-23.2)$ & $<0.0001$ \\
\hline
\end{tabular}

Table 1. Baseline characteristics of study participants. Abbreviations: LDL, low-density lipoprotein; HDL, high-density lipoprotein. ${ }^{a}$ More than once a month during the past year, excluding heavy ( $>30 \mathrm{~g} /$ day) drinkers. ${ }^{b}$ Those who exercised for $\geq 5$ occasions per week for 30 minutes per session, or those who participated in strenuous physical activity for $\geq 3$ occasions per week for 20 minutes per session. ${ }^{c}$ At least once during the past year.

of CPI 3 periodontitis in subjects with the lowest and the highest quartiles of FLI were $12.55 \%$ and $28.86 \%$, respectively. In the case of CPI 4 periodontitis, these percentages were $0.69 \%$ and $3.31 \%$, respectively. In the diabetes subgroup, percentages of CPI 3 periodontitis in subjects with the lowest and highest quartiles of FLI were $36.05 \%$ and $46.12 \%$, respectively. Those of CPI 4 periodontitis were $0 \%$ and $4.48 \%$ respectively.

\section{Discussion}

This study used nationally representative data to determine the association of FLI with periodontitis prevalence in a large probability sample of Korean adults. As FLI increased, the prevalence of periodontitis also increased in multiple logistic regression analysis after adjusting for various previously reported confounding factors for periodontitis. Higher ORs for periodontitis were observed in the diabetes mellitus subgroup in FLI high individuals (3rd and 4th quartiles). Additionally, a more severe form of periodontitis was associated with increasing FLI and the presence of diabetes.

FLI was established as a formula to predict NAFLD, a health problem with a close relationship with obesity. NAFLD has been found in $5-30 \%$ of the general Western population ${ }^{10}$. Surprisingly, it has been found in $90 \%$ of obese population ${ }^{26}$. There is a higher prevalence of NAFLD in patients with chronic diseases such as hypertension, diabetes, and coronary artery atherosclerotic disease that are known to be obesity-related ${ }^{27}$. NAFLD is also associated with periodontitis ${ }^{8}$. In line with these previous findings, FLI showed significant association with periodontitis prevalence in our large probability sample.

Previous reports have utilized FLI to identify NAFLD, especially in extensive epidemiologic studies ${ }^{28,29}$. The most accurate method of hepatic steatosis measurement is magnetic resonance spectroscopy. FLI has been recently shown to have a comparable accuracy to magnetic resonance spectroscopy in predicting the presence of NAFLD ${ }^{30}$. It is important to note that obesity-associated outcomes are apparent in Asian population at a lower level of body fat accumulation. Indeed, it is proposed that mean FLI was lower and FLI was less accurate 


\begin{tabular}{|c|c|c|c|}
\hline & \multicolumn{2}{|c|}{ Periodontitis } & \multirow[b]{3}{*}{$\mathbf{P}$} \\
\hline & No & Yes & \\
\hline & $\mathrm{n}=3159$ & $\mathrm{n}=1113$ & \\
\hline Fatty Liver Index & & & $<0.0001$ \\
\hline 1st quartile & $31(1)$ & $15.9(1.3)$ & \\
\hline 2nd quartile & $25.9(1)$ & $22.2(1.7)$ & \\
\hline 3rd quartile & $21.8(0.9)$ & $27.8(1.7)$ & \\
\hline 4th quartile & $21.4(1)$ & $34.1(1.9)$ & \\
\hline Body mass index & & & $<0.0001$ \\
\hline$<18.5 \mathrm{~kg} / \mathrm{m} 2$ & $5.7(0.5)$ & $2.8(0.6)$ & \\
\hline $18.5-23 \mathrm{~kg} / \mathrm{m} 2$ & $45.4(1.2)$ & $34.2(1.7)$ & \\
\hline $23-25 \mathrm{~kg} / \mathrm{m} 2$ & $20.9(0.8)$ & $24.9(1.6)$ & \\
\hline $25-30 \mathrm{~kg} / \mathrm{m} 2$ & $24.5(0.9)$ & $32.9(1.8)$ & \\
\hline$\geq 30 \mathrm{~kg} / \mathrm{m} 2$ & $3.5(0.4)$ & $5.2(0.9)$ & \\
\hline Waist circumference $(\mathrm{MS}+)^{\mathrm{a}}$ & $27.6(1.1)$ & $40.4(2.4)$ & $<0.0001$ \\
\hline Triglycerides $(\mathrm{MS}+)^{\mathrm{b}}$ & $23.8(0.9)$ & $37.2(1.8)$ & $<0.0001$ \\
\hline Gamma-glutamyl transferase & & & $<0.0001$ \\
\hline 1st quartile & $25.2(1.1)$ & $12.6(1)$ & \\
\hline 2nd quartile & $27.8(1.1)$ & $22.1(1.4)$ & \\
\hline 3rd quartile & $23.8(0.8)$ & $30.2(1.8)$ & \\
\hline 4th quartile & $23.2(1.1)$ & $35(1.8)$ & \\
\hline
\end{tabular}

Table 2. Fatty Liver Index variables of study participants. Values are prevalence and standard error in percentage. Abbreviation: MS, metabolic syndrome. ${ }^{a}$ Waist circumference of $\geq 90 \mathrm{~cm}$ in men and $\geq 80 \mathrm{~cm}$ in women. ${ }^{\mathrm{b}}$ Triglyceride $\geq 150 \mathrm{mg} / \mathrm{dL}$.

\begin{tabular}{|l|l|l|l|l|l|}
\hline \multicolumn{5}{|l|}{ Fatty Liver Index } \\
\cline { 2 - 7 } & 1st quartile & 2nd quartile & 3rd quartile & 4th quartile & P for trend \\
\hline All participants & $1.296(0.978,1.716)$ & $1.481(1.105,1.984)$ & $1.737(1.317,2.292)$ & $<0.001$ \\
\hline Model 1 & 1 & $1.282(0.971,1.694)$ & $1.441(1.071,1.939)$ & $1.664(1.267,2.185)$ & $<0.001$ \\
\hline Model 2 & 1 & $1.287(0.972,1.706)$ & $1.433(1.063,1.932)$ & $1.632(1.235,2.157)$ & 0.001 \\
\hline Model 3 & 1 & $1.298(0.977,1.724)$ & $1.481(1.095,2.002)$ & $1.545(1.142,2.09)$ & 0.006 \\
\hline Non-diabetes subgroup & $1.276(0.960,1.694)$ & $1.422(1.046,1.935)$ & $1.470(1.090,1.981)$ & 0.015 \\
\hline Model 1 & 1 & $1.284(0.965,1.708)$ & $1.418(1.040,1.935)$ & $1.448(1.068,1.963)$ & 0.023 \\
\hline Model 2 & 1 & $1.136(0.329,3.925)$ & $1.317(0.452,3.837)$ & $2.724(0.954,7.774)$ & 0.007 \\
\hline Model 3 & 1 & $1.15(0.347,3.810)$ & $1.507(0.511,4.444)$ & $2.936(1.069,8.068)$ & 0.003 \\
\hline Diabetes subgroup & $1.096(0.325,3.701)$ & $1.445(0.484,4.315)$ & $2.891(1.010,8.269)$ & 0.004 \\
\hline Model 1 & 1 & 1 &
\end{tabular}

Table 3. Periodontitis prevalence in multiple logistic regression models for Fatty Liver Index. Values are adjusted odds ratio and 95\% confidence interval. Adjustments for Model 1: age and sex; Adjustments for Model 2: Model 1 plus smoking, regular exercise, alcohol consumption, income, and education level; Adjustments for Model 3: Model 2 plus tooth brushing frequency and periodic dental checkup.

in NAFLD prediction in Asian studies ${ }^{31}$. However, in recent large-scale Asian population studies in Chinese, Japanese, and Asian-Americans in the U.S., respectively, FLI was recognized and used as a relatively predictable noninvasive diagnostic tool to study of NAFLD also in Asian populations ${ }^{32-34}$. Regardless of ethnicity, this is the first study to show the association of periodontitis prevalence with FLI to the best of our knowledge.

Periodontitis has been reported as one of common complications of diabetes mellitus classically ${ }^{25}$. Diabetes mellitus also has significant association with NAFLD ${ }^{35}$. Furthermore, a recent article has revealed that FLI is a predictor of diabetes mellitus in a longitudinal study ${ }^{36}$. In concordance, our data showed a stronger association between FLI and periodontitis prevalence in the subgroup with diabetes mellitus. The severity of periodontitis was also associated with diabetes, with higher CPI 4 periodontitis percentage in the subgroup of diabetes.

Whether the presence of periodontitis that leads to high FLI status or high FLI plays a role in the development of periodontitis remains unanswered. The interacting role of diabetes with FLI and periodontitis also remains unknown. It is an intrinsic limitation of a cross-sectional analysis such as ours. Although proinflammatory cytokine levels were unavailable to us, other researchers have already provided scientific evidences that the level of proinflammatory cytokines and reactive oxygen species are increased systemically due to periodontal pathogenic 


\begin{tabular}{|l|l|l|l|l|l|}
\hline \multicolumn{7}{|l|}{ Fatty Liver Index } \\
\cline { 2 - 6 } & 1st quartile & 2nd quartile & 3rd quartile & 4th quartile & P \\
\hline All participants & $12.55(1.32)$ & $19.65(1.58)$ & $25.21(1.97)$ & $28.89(1.92)$ & $<0.0001$ \\
\hline CPI 3 periodontitis & $0.69(0.29)$ & $0.73(0.21)$ & $2.34(0.64)$ & $3.32(0.97)$ & $<0.0001$ \\
\hline CPI 4 periodontitis & Non-diabetes subgroup \\
\hline CPI 3 periodontitis & $11.94(1.27)$ & $18.81(1.58)$ & $23.79(2.07)$ & $25.40(2.08)$ & $<0.0001$ \\
\hline CPI 4 periodontitis & $0.71(0.3)$ & $0.67(0.21)$ & $2.46(0.7)$ & $3.08(0.91)$ & $<0.0001$ \\
\hline Diabetes subgroup & $36.06(10.82)$ & $39.67(8.31)$ & $40.11(5.72)$ & $46.12(4.89)$ & 0.7254 \\
\hline CPI 3 periodontitis & $2.23(1.63)$ & $1.09(0.79)$ & $4.48(1.88)$ & \\
\hline CPI 4 periodontitis & &
\end{tabular}

Table 4. Periodontitis prevalence according to the severity of periodontitis. Values are prevalence and standard error in percentage. Abbreviation: CPI, Community Periodontitis Index. CPI 3 is given for cases with a shallow pocket (depth of $3.5-5.5 \mathrm{~mm}$ ) from at least one site in one of the index teeth and CPI 4 is given for cases with a deep pocket (depth $\geq 5.5 \mathrm{~mm}$ ).

biofilms in periodontitis patients ${ }^{37,38}$. This inflammatory condition may lead to high FLI. On the contrary, already high FLI due to obesity-related issues may lead to the development of progression of periodontitis. This was not conclusive with our data. Interestingly, it has been recently proposed that there is a two-way relationship between diabetes mellitus and periodontitis through epidemiological and animal studies ${ }^{18}$. That is, diabetes is a risk factor for increased prevalence and severity of periodontitis, and vice versa.

In conclusion, the findings from this nationally representative probability sample of the Korean population support the hypothesis that FLI may be associated with periodontitis prevalence, especially in subjects with diabetes. To improve public health, active screening of periodontitis in FLI-high population may be clinically beneficial, especially in those with diabetes mellitus.

Received: 11 September 2019; Accepted: 14 February 2020;

Published online: 02 March 2020

\section{References}

1. Meyle, J. \& Chapple, I. Molecular aspects of the pathogenesis of periodontitis. Periodontol. 2000. 69, 7-17 (2015).

2. Eke, P. I., Dye, B. A., Wei, L., Thornton-Evans, G. O. \& Genco, R. J. Prevalence of periodontitis in adults in the United States: 2009 and 2010. J. Dent. Res. 91, 914-920 (2012).

3. Martinez-Herrera, M., Silvestre-Rangil, J. \& Silvestre, F. J. Association between obesity and periodontal disease. A systematic review of epidemiological studies and controlled clinical trials. Med. Oral. Patol. Oral Cir. Bucal. 22, e708-e715 (2017).

4. Humphrey, L. L., Fu, R., Buckley, D. I., Freeman, M. \& Helfand, M. Periodontal disease and coronary heart disease incidence: a systematic review and meta-analysis. J. Gen. Intern. Med. 23, 2079-2086 (2008).

5. Leira, Y. et al. Association between periodontitis and ischemic stroke: a systematic review and meta-analysis. Eur. J. Epidemiol. 32, 43-53 (2017).

6. Lamster, I. B. \& Pagan, M. Periodontal disease and the metabolic syndrome. Int. Dent. J. 67, 67-77 (2017).

7. Preshaw, P. M. et al. Periodontitis and diabetes: a two-way relationship. Diabetologia. 55, 21-31 (2012).

8. Akinkugbe, A. A. et al. Periodontitis and Non-alcoholic Fatty Liver Disease, a population-based cohort investigation in the Study of Health in Pomerania. J. Clin. Periodontol. 44, 1077-1087 (2017).

9. Bedogni, G. et al. The Fatty Liver Index: a simple and accurate predictor of hepatic steatosis in the general population. BMC Gastroenterol. 6, 33 (2006).

10. Benedict, M. \& Zhang, X. Non-alcoholic fatty liver disease: An expanded review. World J. Hepatol. 9, 715-732 (2017).

11. Choi, D. H. et al. Usefulness of the Waist Circumference-to-Height Ratio in Screening for Obesity and Metabolic Syndrome among Korean Children and Adolescents: Korea National Health and Nutrition Examination Survey, 2010-2014. Nutrients. 9 (2017).

12. Pan, Y. et al. Curcumin improves glycolipid metabolism through regulating peroxisome proliferator activated receptor gamma signalling pathway in high-fat diet-induced obese mice and 3T3-L1 adipocytes. R. Soc. Open. Sci. 4, 170917 (2017).

13. Han, K., Park, Y. M. \& Park, J. B. Evaluation of an association between long sleep duration and periodontal disease among men and women using nationally representative data. Gac. Sanit. 32, 143-150 (2018).

14. Song, S. J., Han, K., Lee, S. S. \& Park, J. B. Association between the number of natural teeth and diabetic retinopathy among type 2 diabetes mellitus: The Korea national health and nutrition examination survey. Med. 96, e8694 (2017).

15. Ban, M. J. et al. Korean survey data reveals an association of chronic laryngitis with tinnitus in men. PLoS One. 13, e0191148 (2018).

16. Alberti, K. G. et al. Harmonizing the metabolic syndrome: a joint interim statement of the International Diabetes Federation Task Force on Epidemiology and Prevention; National Heart, Lung, and Blood Institute; American Heart Association; World Heart Federation; International Atherosclerosis Society; and International Association for the Study of Obesity. Circulation. 120, 1640-1645 (2009).

17. Sarwar, N. et al. Diabetes mellitus, fasting blood glucose concentration, and risk of vascular disease: a collaborative meta-analysis of 102 prospective studies. Lancet. 375, 2215-2222 (2010).

18. Zhou, X., Zhang, W., Liu, X., Zhang, W. \& Li, Y. Interrelationship between diabetes and periodontitis: role of hyperlipidemia. Arch. Oral. Biol. 60, 667-674 (2015).

19. Pischon, N. et al. Obesity, inflammation, and periodontal disease. J. Dent. Res. 86, 400-409 (2007).

20. Abbasalizad Farhangi, M., Nikniaz, L. \& Nikniaz, Z. Higher dietary acid load potentially increases serum triglyceride and obesity prevalence in adults: An updated systematic review and meta-analysis. PLoS One. 14, e0216547 (2019).

21. Whitfield, J. B. Gamma glutamyl transferase. Crit. Rev. Clin. Lab. Sci. 38, 263-355 (2001).

22. Coku, V. \& Shkembi, X. Serum Gamma-glutamyltransferase and Obesity: is there a Link? Med. Arch. 72, 112-115 (2018).

23. Han, K. \& Park, J. B. Clinical implications of age and sex in the prevalence of periodontitis in Korean adults with diabetes. Exp. Ther. Med. 15, 3865-3873 (2018). 
24. Han, K. et al. Association of Periodontitis With Urinary Albumin Excretion in Korean Adults With Diabetes: The 2012 Korea National Health and Nutrition Examination Survey. Med. 94, e1839 (2015).

25. Loe, H. Periodontal disease. The sixth complication of diabetes mellitus. Diabetes Care. 16, 329-334 (1993).

26. Tai, F. W., Syn, W. K. \& Alazawi, W. Practical approach to non-alcoholic fatty liver disease in patients with diabetes. Diabet. Med. 32, $1121-1133$ (2015).

27. Jiang, Z. Y. et al. Fatty liver index correlates with non-alcoholic fatty liver disease, but not with newly diagnosed coronary artery atherosclerotic disease in Chinese patients. BMC Gastroenterol. 13, 110 (2013).

28. Koehler, E. M. et al. External validation of the fatty liver index for identifying nonalcoholic fatty liver disease in a population-based study. Clin. Gastroenterol. Hepatol. 11, 1201-1204 (2013).

29. Meffert, P. J. et al. Development, external validation, and comparative assessment of a new diagnostic score for hepatic steatosis. Am. J. Gastroenterol. 109, 1404-1414 (2014).

30. Cuthbertson, D. J. et al. External validation of the fatty liver index and lipid accumulation product indices, using $1 \mathrm{H}$-magnetic resonance spectroscopy, to identify hepatic steatosis in healthy controls and obese, insulin-resistant individuals. Eur. J. Endocrinol. 171, 561-569 (2014).

31. Kim, J. H., Kwon, S. Y., Lee, S. W. \& Lee, C. H. Validation of fatty liver index and lipid accumulation product for predicting fatty liver in Korean population. Liver Int. 31, 1600-1601 (2011).

32. Li, C. et al. Both WHR and FLI as Better Algorithms for Both Lean and Overweight/Obese NAFLD in a Chinese Population. J. Clin. Gastroenterol. 53, e253-e260 (2019).

33. Tabuchi, H., Maegawa, H., Tobe, K., Nakamura, I. \& Uno, S. Effect of ipragliflozin on liver function in Japanese type 2 diabetes mellitus patients: a subgroup analysis of the STELLA-LONG TERM study (3-month interim results). Endocr. J. 66, 31-41 (2019).

34. Golabi, P. et al. Prevalence and outcomes of non-alcoholic fatty liver disease (NAFLD) among Asian American adults in the United States. Liver Int. 39, 748-757 (2019).

35. Leite, N. C., Villela-Nogueira, C. A., Cardoso, C. R. \& Salles, G. F. Non-alcoholic fatty liver disease and diabetes: from physiopathological interplay to diagnosis and treatment. World J. Gastroenterol. 20, 8377-8392 (2014).

36. Franch-Nadal, J. et al. Fatty liver index is a predictor of incident diabetes in patients with prediabetes: The PREDAPS study. PLoS One. 13, e0198327 (2018).

37. Könönen, E., Gursoy, M. \& Gursoy, U. K. Periodontitis: A Multifaceted Disease of Tooth-Supporting Tissues. J. Clin. Medicine. 8, 1135 (2019).

38. Polak, D. \& Shapira, L. An update on the evidence for pathogenic mechanisms that may link periodontitis and diabetes. J. Clin. Periodontol. 45, 150-166 (2018).

\section{Acknowledgements}

This research was supported by a grant of the Korea Health Technology R\&D Project through the Korea Health Industry Development Institute (KHIDI) funded by the Ministry of Health \& Welfare, Republic of Korea (grant number: HI18C0275). The funder had no role in study design, data collection, data analysis, decision to publish, or preparation of the manuscript.

\section{Author contributions}

Conceptualization, J.K., K.H. and S.K.; Data curation, Y.P., Y.A. and K.H.; Funding acquisition, S.K.; Methodology, G.L. and K.H.; Writing - original draft, J.K.; Writing - review \& editing, H.S., Y.P., Y.A., K.H. and S.K.

\section{Competing interests}

The authors declare no competing interests.

\section{Additional information}

Supplementary information is available for this paper at https://doi.org/10.1038/s41598-020-60797-7.

Correspondence and requests for materials should be addressed to K.H. or S.-H.K.

Reprints and permissions information is available at www.nature.com/reprints.

Publisher's note Springer Nature remains neutral with regard to jurisdictional claims in published maps and institutional affiliations.

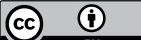

Open Access This article is licensed under a Creative Commons Attribution 4.0 International

License, which permits use, sharing, adaptation, distribution and reproduction in any medium or format, as long as you give appropriate credit to the original author(s) and the source, provide a link to the Creative Commons license, and indicate if changes were made. The images or other third party material in this article are included in the article's Creative Commons license, unless indicated otherwise in a credit line to the material. If material is not included in the article's Creative Commons license and your intended use is not permitted by statutory regulation or exceeds the permitted use, you will need to obtain permission directly from the copyright holder. To view a copy of this license, visit http://creativecommons.org/licenses/by/4.0/.

(c) The Author(s) 2020 